\title{
ANALYSIS OF BROADBAND GEVD-BASED BLIND SOURCE SEPARATION
}

\author{
Soydan Redif \\ Dept. of Electrical and Electronics Engineering, \\ European University of Lefke, \\ Northern Cyprus,TR-10 Mersin,Turkey. \\ sredif@eul.edu.tr
}

\author{
Jen Pestana*, Ian K. Proudler ${ }^{\dagger}$ \\ University of Strathclyde, \\ Glasgow G1 1XW, Scotland, UK \\ jennifer.pestana@strath.ac.uk \\ ian.proudler@strath.ac.uk
}

\begin{abstract}
One approach to blind source separation of instantaneously mixed, non-stationary sources involves using the generalized eigenvalue decomposition of two estimated covariance matrices. The assumption is that the source statistics change with time whilst the mixing matrix does not. A recent generalisation of this approach to convolutive mixtures was achieved by extending the generalized eigenvalue decomposition to polynomial matrices. In this paper, we present a further investigation into this broadband BSS technique. We derive some expressions for the conditions under which source separation is possible. The validity of our analysis is illustrated through some computer simulations.
\end{abstract}

Index Terms - non-stationary, broadband, blind signal separation, generalised eigenvalue decomposition.

\section{INTRODUCTION}

Blind source separation (BSS) is the recovery of source signals from measurements of mixtures of the signals without any prior knowledge of the signals or mixing process. Much effort has been devoted to developing algorithms for instantaneous BSS and convolutive (broadband) BSS using statistical information about the source signals. In the case of instantaneously mixed, non-stationary signals, one approach uses joint diagonalization of two "target matrices" in order to estimate the unknown, fixed mixing matrix $[1,2,3]$. These (covariance) matrices are usually calculated over different time-intervals so as to capture the non-stationarity of the sources. The joint diagonalization is achieved using the generalized eigenvalue decomposition (GEVD). This algorithm is for narrowband signals and so would generally not work if the sources underwent convolutive mixing. Generalisations of the GEVD to the polynomial domain, namely polynomial

\footnotetext{
* Department of Mathematics

${ }^{\dagger}$ Centre for Signal \& Image Processing (CeSIP), Dept. of Electronic \& Electrical Engineering. This work was supported by the Engineering and Physical Sciences Research Council (EPSRC) Grant number EP/S000631/1 and the MOD University Defence Research Collaboration (UDRC) in Signal Processing.
}

GEVD (PGEVD), have recently been proposed $[4,5]$. They are based on recent work on extending the eigenvalue decomposition (EVD) to polynomial matrices, or polynomial eigenvalue decomposition (PEVD) [6,7]. Note that, strictly speaking, these algorithms use Laurent polynomials. Recently Weiss $[8,9]$ has explored the conditions under which a PEVD exists and has shown that, in general, the decomposition requires Laurent series. In this paper, we investigate further this PGEVD-based broadband BSS approach and look at conditions under which the source signals can be recovered. First we review the (scalar) GEVD and then look the conditions under which a PGEVD exists. We then study the conditions under which the BSS problem can be solved. Finally the results of a simple computer simulation are presented. We use the notation $u(z)$ to represent a function $u: \mathbb{C} \rightarrow \mathbb{C}$. If a variable has other relevant properties (e.g. analyticity; a Laurent polynomial) it is mentioned in the text. Bold face variables are matrices.

\section{GENERALISED EIGENVALUE DECOMPOSITION}

The scalar GEVD of the two matrices $\{A, B\} \in \mathbb{C}^{M \times M}$ can be written:

$$
A U=B U \Lambda
$$

where the matrix $U \in \mathbb{C}^{M \times M}$ contains the generalised eigenvectors and $\Lambda \in \mathbb{C}^{M \times M}$ is a diagonal matrix containing the generalised eigenvalues. The scalar GEVD is not well defined if both matrices are rank deficient [10]. Hence, without loss of generality, we assume that $B$ is invertible.

Generalising eqn. (1), the GEVD of two Laurent polynomial matrices $\boldsymbol{A}(z)$ and $\boldsymbol{B}(z)$ is written as:

$$
\boldsymbol{A}(z) \boldsymbol{U}(z)=\boldsymbol{B}(z) \boldsymbol{U}(z) \boldsymbol{\Lambda}(z)
$$

where $\{\boldsymbol{A}(z), \boldsymbol{B}(z), \boldsymbol{U}(z), \boldsymbol{\Lambda}(z)\}: \mathbb{C} \rightarrow \mathbb{C}^{M \times M}$. Note that for a specific value of $z$, eqn. (2) is just the scalar GEVD. Hence, as above, we assume, that $\boldsymbol{B}(z)$ is invertible. So far there seems to be no published existence proof for a Laurent series GEVD. However the existence of the Laurent series EVD of a parahermitian matrix has been studied [11, 12, 
$13,8,9]$ and it is known that the GEVD can be cast as the EVD of a parahermitian matrix (cf. see eqn.(6-8) of [4]). Specifically, since $\boldsymbol{B}(z)$ is parahermitian, invertible and analytic its $\operatorname{EVD}^{1}$ is $\boldsymbol{B}(z)=\boldsymbol{Q}(z) \boldsymbol{\Lambda}_{B}(z) \boldsymbol{Q}^{P}(z)$, at least on the unit circle. Also, $\boldsymbol{\Lambda}_{B}(z)$ is invertible on the unit circle and admits an analytic square-root there, and so $C(z)=$ $\left\{\boldsymbol{L}^{-1}(z)\right\}^{P} \boldsymbol{A}(z) \boldsymbol{L}^{-1}(z)$, where $\boldsymbol{L}(z)=\boldsymbol{\Lambda}_{B}^{1 / 2}(z) \boldsymbol{Q}^{P}(z)$, is parahermitian, and is analytic on the unit circle. Thus, on the unit circle eqn.(2) becomes:

$$
\boldsymbol{C}(z) \boldsymbol{V}(z)=\boldsymbol{V}(z) \boldsymbol{\Lambda}(z)
$$

which is a Laurent series EVD with $\boldsymbol{V}(z)=\boldsymbol{L}(z) \boldsymbol{U}(z)$. Thus we may evoke the results of Weiss et. al $[8,9]$ to infer the existence of the generalised eigenvalues $\boldsymbol{\Lambda}(z)$ and $\boldsymbol{V}(z)$. The existence of the matrices $\boldsymbol{V}(z)$ and $\boldsymbol{L}^{-1}(z)$ imply the existence of the generalised eigenvectors $\boldsymbol{U}(z)$.

The results of Weiss et. al $[8,9]$ show that the generalised eigenvalues in the matrix $\Lambda(z)$ exist as unique and convergent but likely infinite-length Laurent series provided they are Hölder continuous on the unit circle. The columns of the matrix $\boldsymbol{V}(z)$ can be multiplied by an arbitrary phase response that is not defined by the matrix $C(z)$. If the generalised eigenvalues are selected as analytic functions on the unit circle, and if the phase response is selected such that the elements of $\boldsymbol{V}(z)$ are Hölder continuous with $\alpha>1$ on the unit circle, then $\boldsymbol{V}(z)$ exists as a convergent Laurent series. If the generalised eigenvalues are analytic but the phase response is discontinuous or if the eigenvalues are not analytic, $\boldsymbol{V}(z)$ does not have an absolutely convergent Laurent series solution but can generally be approximated by Laurent polynomials. Analytic eigenvalues do not exist if a paraunitary similarity transformation exists that brings $\boldsymbol{C}(z)$ into a form with pseudo-circulant blocks on the diagonal with at least one of a dimension greater than one. The generalised eigenvectors are given by $\boldsymbol{U}(z)=\boldsymbol{L}^{-1}(z) \boldsymbol{V}(z)$. Since $\boldsymbol{B}(z)$ is analytic so is $\boldsymbol{L}(z)$. Thus the generalised eigenvectors $\boldsymbol{U}(z)$ can exist as absolutely convergent Laurent series or can be approximated by Laurent polynomials.

\subsection{Integral Domains}

In the absence of some constraints, the algebra of Laurent series is a ring. Thus there could be divisors of zero. This is problematic because, as we shall see, we will be faced with equations of the form $x y=0$ where $x \neq 0$ and from which we wish to infer that $y=0$. However, integral domains are rings which have no divisors of zero and analytic functions form an integral domain (see, e.g., p.127 of Freitag [15]). In the following we therefore will require our functions to be analytic.

\footnotetext{
${ }^{1}$ Here $\boldsymbol{A}^{P}(z)$ is the paraconjugate of $\boldsymbol{A}(z): \boldsymbol{A}^{P}(z)=\left[\mathbf{A}\left(1 / z^{*}\right)\right]^{\mathrm{H}}[14]$ i.e. the coefficients are conjugated and $z$ is replaced by $1 / z$.
}

\section{NON-STATIONARY BLIND SIGNAL SEPARATION}

\subsection{Signal Model and GEVD}

We assume $N$ independent sources transmitting signals $s_{k}$ $1 \leq k \leq N$ that propagate through some channels $H_{i, j}(z)$ $1 \leq j \leq M$ to an $M$-element array. Further, we assume that two datasets are collected at different times and that the mixing matrix has not changed but the signal statistics have. The received signals can be expressed as

$$
\boldsymbol{x}_{i}(z)=\boldsymbol{s}_{i}(z) \boldsymbol{H}(z) \quad 1 \leq i \leq 2 .
$$

The associated space-time covariance matrices are thus

$$
\boldsymbol{R}_{i}(z)=\boldsymbol{H}^{P}(z) \boldsymbol{S}_{i}(z) \boldsymbol{H}(z) \quad 1 \leq i \leq 2
$$

where $S_{i}(z): \mathbb{C} \rightarrow \mathbb{C}^{N \times N}$ is the cross spectral density (CSD) matrix for the $i$-th dataset and is diagonal by virtue of the independence of the source signals. In the following, we look at the connection between the GEVD of the systems $\left(\boldsymbol{R}_{1}(z), \boldsymbol{R}_{2}(z)\right)$ and $\left(\boldsymbol{S}_{1}(z), \boldsymbol{S}_{2}(z)\right)$.

Assume we have calculated a Laurent series GEVD of $\boldsymbol{R}_{1}(z)$ and $\boldsymbol{R}_{2}(z)$. This implies that $\boldsymbol{R}_{2}(z)$ must be full rank (as per section 2). Hence both $\boldsymbol{H}(z)$ and $\boldsymbol{S}_{2}(z)$ are full rank (cf. eqn. (5)). This in turn implies that $M \leq N$ i.e. there must be at least the same number of signal sources as sensors (or else $\boldsymbol{H}(z)$ would not be full rank). If $M<N$ we have an under-determined BSS problem and we will not be able to recover the signals without extra information. Hence in the following we assume $M=N$.

Taking the Laurent series GEVD of $\boldsymbol{R}_{1}(z)$ and $\boldsymbol{R}_{2}(z)$ we may write, for some $k \in\{1: M\}$,

$$
\boldsymbol{R}_{1}(z) \boldsymbol{u}_{k}(z)=\lambda_{k}(z) \boldsymbol{R}_{2}(z) \boldsymbol{u}_{k}(z) .
$$

Since $\boldsymbol{u}_{k}(z)$ is an eigenvector, it is non-zero so

$$
\operatorname{det}\left(\boldsymbol{R}_{1}(z)-\lambda_{k}(z) \boldsymbol{R}_{2}(z)\right)=0 .
$$

Now using eqn. (5), after some algebra, we find

$$
\operatorname{det}(\boldsymbol{H}(z))^{2} \operatorname{det}\left(\boldsymbol{S}_{1}(z)-\lambda_{k}(z) \boldsymbol{S}_{2}(z)\right)=0 .
$$

Since $\boldsymbol{H}(z)$ is full rank, $\operatorname{det}(\boldsymbol{H}(z)) \neq 0$ and so

$$
\operatorname{det}\left(\left(\boldsymbol{S}_{1}(z)-\lambda_{k}(z) \boldsymbol{S}_{2}(z)\right)\right)=0 .
$$

Hence the generalised eigenvalues of $\left(\boldsymbol{R}_{1}(z), \boldsymbol{R}_{2}(z)\right)$ are also generalised eigenvalues of $\left(\boldsymbol{S}_{1}(z), \boldsymbol{S}_{2}(z)\right)$. Furthermore let $\boldsymbol{w}_{k}(z)$ be the generalised eigenvector for $\left(\boldsymbol{S}_{1}(z), \boldsymbol{S}_{2}(z)\right)$ corresponding to $\lambda_{k}(z)$ so that

$$
\left(\boldsymbol{S}_{1}(z)-\lambda_{k}(z) \boldsymbol{S}_{2}(z)\right) \boldsymbol{w}_{k}(z)=0 .
$$

Now, $\boldsymbol{H}(z)$ is full rank so its inverse exists and $\boldsymbol{S}_{i}(z)$ can be written in terms of $\boldsymbol{R}_{i}(z)(1 \leq i \leq 2)$ (cf. eqn. (5)). Inserting this into eqn. (10) we get, after some algebra,

$$
\left(\boldsymbol{R}_{1}(z)-\lambda_{k}(z) \boldsymbol{R}_{2}(z)\right) \boldsymbol{H}^{-1}(z) \boldsymbol{w}_{k}(z)=0
$$


thus $\boldsymbol{H}^{-1}(z) \boldsymbol{w}_{k}(z)$ is the generalised eigenvector of the system $\left(\boldsymbol{R}_{1}(z), \boldsymbol{R}_{2}(z)\right)$ corresponding to $\lambda_{k}(z)$. Thus

$$
\boldsymbol{U}(z)=\boldsymbol{H}^{-1}(z) \boldsymbol{W}(z) .
$$

Hence the generalised eigenvalues of $\left(\boldsymbol{R}_{1}(z), \boldsymbol{R}_{2}(z)\right)$ are the ratios of the power spectral densities (PSD) of the source signals, and the generalised eigenvectors are related to the generalised eigenvalues of $\left(\boldsymbol{S}_{1}(z), \boldsymbol{S}_{2}(z)\right)$ by the matrix $\boldsymbol{H}(z)$.

\subsection{Signal Recovery}

In the following we show that $\boldsymbol{W}(z)$ can be a permuted diagonal matrix. This leads to the conclusion that $\boldsymbol{U}(z)$ can be used to recover the source signals from the received data.

Since $\boldsymbol{S}_{i}(z) i=1,2$ are diagonal, we have from eqn. (10) that, for $j \in\{1: M\}$

$$
\left(S_{1, j}(z)-\lambda_{k}(z) S_{2, j}(z)\right) w_{k, j}(z)=0
$$

We assume that all the functions in eqn. (13) are analytic, at least on the unit circle. In this case (see section 2.1), either $\left(S_{1, j}(z)-\lambda_{k}(z) S_{2, j}(z)\right) \equiv 0$ or $w_{k, j}(z) \equiv 0$ in the domain of analyticity (which includes the unit circle). For every fixed $z=\mathrm{e}^{\mathrm{j} \Omega_{0}}$ eqn. (10) is a Hermitian definite scalar GEVD, $w_{k}\left(\mathrm{e}^{\mathrm{j} \Omega_{0}}\right)$ is not the zero vector and at least one element, say the $j_{0}$-th, is non-zero. Although it is possible that $w_{k, j_{0}}\left(\mathrm{e}^{\mathrm{j} \Omega}\right)$ is zero at some other value $\Omega_{1}$, since it is analytic, it can only be zero at isolated points. It follows that

$$
S_{1, j_{0}}(z)-\lambda_{k}(z) S_{2, j_{0}}(z) \equiv 0 .
$$

Thus, there must be at least one index $j_{0}$ such that eqn. (14) holds. As $\boldsymbol{S}_{2}(z)$ is analytic, full rank, and diagonal, we therefore have:

$$
\lambda_{k}(z)=S_{1, j_{0}}(z) / S_{2, j_{0}}(z)
$$

i.e. the generalized eigenvalues are the ratio of the source PSDs. If, in addition, there is another index $j_{1} \neq j_{0}$ such that $w_{k, j_{1}}(z)$ is also non-zero, then $S_{1, j_{1}}(z)-\lambda_{k}(z) S_{2, j_{1}}(z) \equiv$ 0 and we have $\lambda_{k}(z)=S_{1, j_{1}}(z) / S_{2, j_{1}}(z)$. From eqn. (15) we see that two of the PSD ratios are equal, and $\lambda_{k}(z)$ is an eigenvalue with algebraic multiplicity greater than one.

If we assume, for the moment, that there are no identical PSD ratios, then only $w_{k, j_{0}}(z)$ is nonzero i.e.

$$
w_{k, j}(z)=0 \quad j \neq j_{0}
$$

and the rows of the matrix $\boldsymbol{W}(z)$ contain one non-zero element (the $j_{0}$-th one). Now consider the row $m \neq k$. We have

$$
\left(\boldsymbol{S}_{1}(z)-\lambda_{m}(z) \boldsymbol{S}_{2}(z)\right) \boldsymbol{w}_{m}(z)=0 .
$$

As we are assuming that the generalised eigenvalues all have an algebraic multiplicity of one, the vector $\boldsymbol{w}_{m}(z)$ contains only one non-zero element (cf. eqn. (16)). Assume it is also the $j_{0}$-th one. Then eqn. (17) gives us

$$
\lambda_{m}(z)=S_{1, j_{0}}(z) / S_{2, j_{0}}(z)=\lambda_{k}(z) .
$$

But by assumption $\lambda_{m}(z) \neq \lambda_{k}(z)$ hence the non-zero element of the vector $\boldsymbol{w}_{m}(z)$ cannot be the $j_{0}$-th one. Thus the columns of the matrix $\boldsymbol{W}(z)$ contain only one non-zero element. Hence the matrix of generalized eigenvectors can be written as

$$
\boldsymbol{W}(z)=\boldsymbol{D}(z) \Pi
$$

where $\boldsymbol{D}(z)$ contains the non-zero elements of the matrix $\boldsymbol{W}(z)$ on its diagonal and $\Pi$ is a permutation. Thus, using eqns. (4) and (12), we have

$$
\boldsymbol{x}(z) \boldsymbol{U}(z)=\boldsymbol{s}(z) \boldsymbol{D}(z) \Pi
$$

and, up to scaling and permutations, we have recovered the source signals from the received data.

On the other hand if we have eigenvalues with an algebraic multiplicity greater than one (i.e. equal PSD ratios) then the rows of the matrix $\boldsymbol{W}(z)$ contain more than one non-zero element. The number of non-zero elements will be equal to the algebraic multiplicity of the corresponding eigenvalue. Furthermore the columns of the matrix $\boldsymbol{W}(z)$ will also contain the same number of non-zero elements. Hence the matrix of generalized eigenvectors can be written as $\boldsymbol{W}(z)=\boldsymbol{\Delta}(z) \Pi$ where $\boldsymbol{\Delta}(z)$ is a block-diagonal matrix that contains the non-zero elements of the matrix $\boldsymbol{W}(z)$ and $\Pi$ is a permutation. Taking the Laurent series SVD of the blocks of $\boldsymbol{\Delta}(z)$, we see the effect of $\boldsymbol{W}(z)$ (cf. eqn. (20)) is to apply a parunitary mixing to the signals that have identical PSD ratios as well as scaling and permuting them.

\section{SIMULATIONS}

The two published algorithms for computing a 'broadband' GEVD [4, 5] use the ideas behind a PEVD algorithm [7]. The latter tends to produce eigenvalues that are spectrally majorised [16] and so are only analytic in special cases, and hence violate the requirements derived in section 2. Clearly, if the source PSD ratios are majorised, these two algorithms would work (e.g. see [5]). In the absence of a genuine Laurent series GEVD algorithm, the simulations below are based on a pseudo-algorithm which requires human intervention: The PGEVD algorithm in [5] is first applied directly to the two matrices $\boldsymbol{R}_{1}(z)$ and $\boldsymbol{R}_{2}(z)$. The polynomial matrix of majorised eigenvalues output from this algorithm is then transformed to the frequency domain. From a plot of the magnitude of the eigenvalues, the permutation that makes the eigenvalues a smooth function of frequency is determined; this is applied to the eigenvalues and eigenvectors which are then transformed to the time domain producing $\boldsymbol{U}(z)$ and $\boldsymbol{\Lambda}(z)$.

Due to lack of space, we only present results for two experiments. Using an invertible, FIR mixing matrix $\boldsymbol{A}$, of order 3 , we generate two sets of four convolutively mixed signals with corresponding CSD matrices $\boldsymbol{R}_{1}(z)$ and $\boldsymbol{R}_{2}(z)$. In the first experiment, the CSD matrices are generated from 


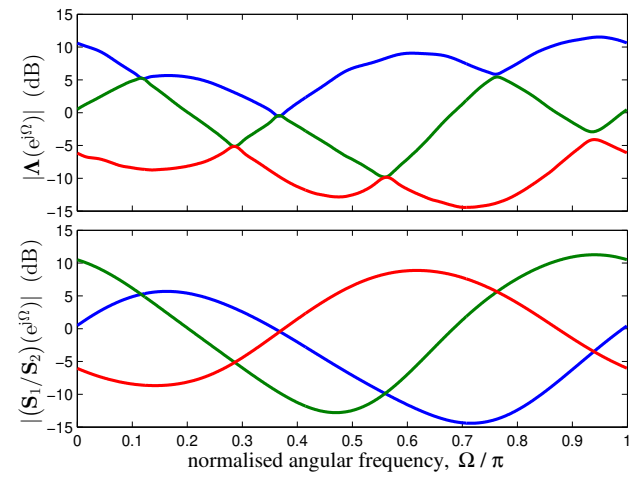

(a) Majorised Generalised Eigenvalues $\boldsymbol{\Lambda}(z)$ (top) and PSD Ratios $\boldsymbol{S}_{1}(z) / \boldsymbol{S}_{2}(z)$ (bottom).

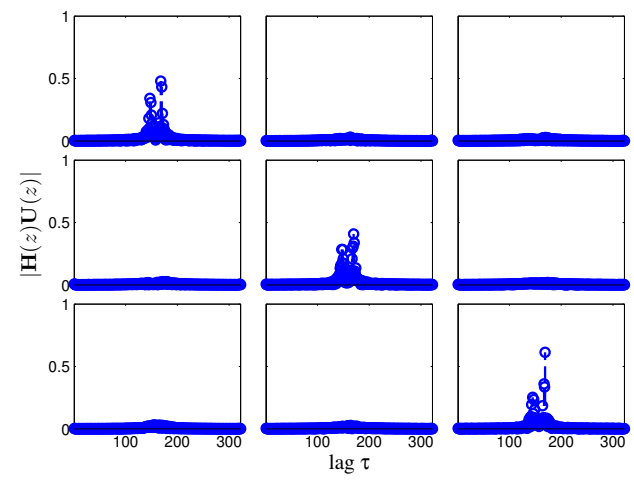

(b) Signal Separation Matrix $\boldsymbol{H}(z) \boldsymbol{U}(z)$.

Fig. 1. Expt. 1: Distinct Spectrally Unmajorised Sources.

the source model of [7] modified such that the sources corresponding to $\boldsymbol{R}_{1}(z)$ are not spectral majorised. This represents conditions in which good signal separation performance should be achievable but which cause problems for the existing PGEVD algorithms (which cannot recover unmajorised signals). In the second experiment, the source model is modified to produce some very similar PSD ratios. This tests the main limitation of the approach.

For experiment 1, the PSD ratios are shown in Fig. 1(a) along with the generalised eigenvalues obtained using the algorithm of Redif [5]. Note how this algorithm majorises the eigenvalues and hence will not recover the source signals. Using the pseudo-Laurent series GEVD algorithm outlined above, we obtained eigenvalues identical to the PSD ratio matrix shown in Fig. 1(a)(bottom). Fig. 1(b) shows the signal separation matrix (i.e. $\boldsymbol{H}(z) \boldsymbol{U}(z)$ cf. eqn.(20)) using the pseudo-Laurent series GEVD algorithm. The separation matrix turns out to be diagonal and shows that the source signals would be successfully recovered (up to a polynomial scaling which is equivalent to a convolution). For experiment 2, the eigenvalues from the pseudo-algorithm and the PSD ratio matrix are shown in Fig. 2(a). We see that the eigenvalues are not

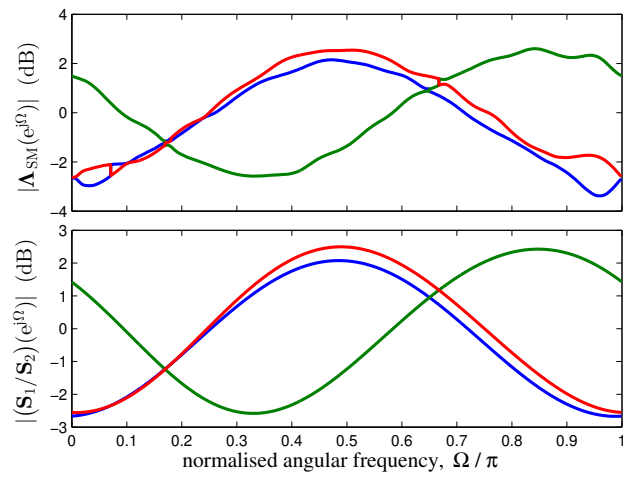

(a) Analytic Generalised Eigenvalues $\boldsymbol{\Lambda}(z)$ (top) and PSD Ratios $\boldsymbol{S}_{1}(z) / \boldsymbol{S}_{2}(z)$ (bottom).

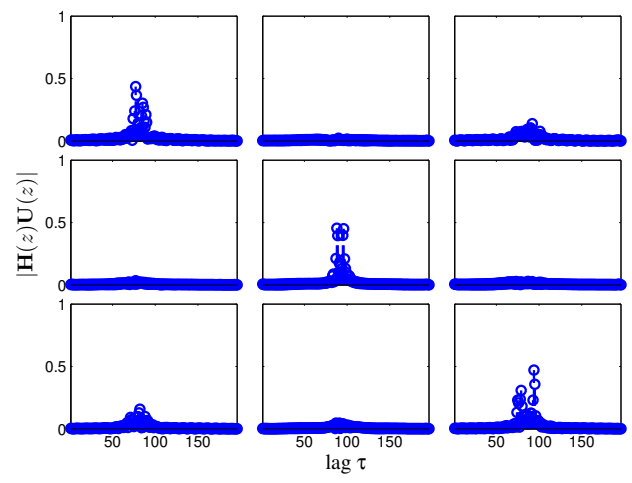

(b) Signal Separation Matrix $\boldsymbol{H}(z) \boldsymbol{U}(z)$.

Fig. 2. Expt. 2: Indistinct Sources.

particularly good estimates of the PSD ratios and hence signal separation is likely to be poor. Fig. 2(b) shows the signal separation matrix which has off-diagonal terms indicating that the signals will not be completely unmixed.

\section{CONCLUSIONS}

We have presented an analysis of a Laurent series GEVD approach to broadband non-stationary BSS. This involves gathering two sets of data such that the signal CSD matrix changes between measurements but the mixing matrix does not. The conditions under which the signals can be separated and uniqueness of the results are given. Specifically if the PSD ratios are analytic on the unit circle; the mixing matrix and the CSD matrix of at least one measurement are full rank; and the matrix $C(z)$ is not similar to a pseudo-circulant; then the source signals can be recovered up to a permutation and a frequency dependent scaling, and possibly a paraunitary mixing. The paraunitary mixing only occurs if the measurements of two or more sources have identical PSD ratios. Recovery of the signals requires an analytic Laurent series GEVD algorithm. 


\section{REFERENCES}

[1] L. Parra and P. Sajda, "Blind source separation via generalized eigenvalue decomposition," J. Mach. Learn. Res., vol. 4, pp. 1261-1269, 2003.

[2] A. Yeredor, "Performance analysis of GEVD-based source separation with second-order statistics," Trans. on Signal Process., vol. 59, pp. 5077-5082, Oct 2011.

[3] A. M. Tomé, "The generalized eigendecomposition approach to the blind source separation problem," Digit. Signal Process., vol. 16, pp. 288-302, May 2006.

[4] J. Corr, J. Pestana, S. Weiss, S. Redif, and M. Moonen, "Investigation of a polynomial matrix generalised EVD for multi-channel Wiener filtering," in 50th Asilomar Conference on Signals, Systems and Computers, Pacific Grove, CA, USA, July 2016, pp. 1354-1358, IEEE.

[5] S. Redif, "Convolutive blind signal separation via polynomial matrix generalized eigenvalue decomposition," Electronic Letters, vol. 53, pp. 87-89, Jan 2017.

[6] J. G. McWhirter, P. D. Baxter, T. Cooper, S. Redif, and J. Foster, "An EVD algorithm for para-hermitian polynomial matrices," Trans. on Signal Process., vol. 55, pp. 2158-2169, May 2007.

[7] S. Redif, S. Weiss, and J. McWhirter, "Sequential matrix diagonalization algorithms for polynomial EVD of parahermitian matrices," Trans. on Signal Process., vol. 63, pp. 81-89, Jan. 2015.

[8] S. Weiss, J. Pestana, and I. K. Proudler, "On the existence and uniqueness of the eigenvalue decomposition of a parahermitian matrix," Trans. on Signal Process., vol. 66, pp. 2659-2672, Mar. 2018.

[9] S. Weiss, F. Coutts, J. Pestana, and I. K. Proudler, "Correction to 'On the existence and uniqueness of the eigenvalue decomposition of a parahermitian matrix'," Trans. on Signal Process., vol. 66, pp. 6325-6327, Dec. 2018.

[10] Z. Bai, T. Ericsson, and T. Kowalski, "Symmetric indefinite Lanczos method (Section 8.6)," in Templates for the Solution of Algebraic Eigenvalue Problems: A Practical Guide, Z. Bai, J. Demmel, J. Dongarra, A. Ruhe, and H. van der Vorst, Eds. SIAM, Philadelphia, 2000.

[11] T. Kato, Die Grundlehren der mathematischen Wissenschaften in Einzeldarstellungen, vol. 132, SpringerVerlag Berlin Heidelberg, 1966.

[12] F. Rellich, "Störungstheorie der spektralzerlegung. I. Mitteilung. Analytische störung der isolierten punkteigenwerte eines beschränkten operators," Mathematische Annalen, vol. 113, pp. DC-DCXIX, 1937.
[13] A. Bunse-Gerstner, R. Byers, V. Mehrmann, and N.K. Nicols, "Numerical computation of an analytic singular value decomposition of a matrix valued function," $\mathrm{Nu}$ mer. Math, vol. 60, pp. 1-40, 1991.

[14] P. P. Vaidyanathan, Multirate Systems and Filter Banks, Prentice Hall, Englewood Cliffs, NJ, 1993.

[15] E. Freitag and R. Busam, Complex Analysis, SpringerVerlag Berlin Heidelberg, 2009.

[16] J.G. McWhirter and Z. Wang, "A novel insight to the SBR2 algorithm fordiagonalising para-hermitian matrices," in 11th IMA Conference on Mathematics in Signal Processing, Birmingham, U.K., Dec. 2016, pp. 1-4. 